\title{
A Role for Lipids in Protein Sorting?
}

\author{
Auxiliadora Aguilera-Romero* and Manuel Muñiz*
}

\begin{abstract}
Lipid and protein diversity provides structural and functional identity to the membrane compartments that define the eukaryotic cell. This compositional heterogeneity is maintained by the secretory pathway, which feeds newly synthesized proteins and lipids to the endomembrane systems. The precise sorting of lipids and proteins through the pathway guarantees the achievement of their correct delivery. Although proteins have been shown to be key for sorting mechanisms, whether and how lipids contribute to this process is still an open discussion. Our laboratory, in collaboration with other groups, has recently addressed the long-postulated role of membrane lipids in protein sorting in the secretory pathway, by investigating in yeast how a special class of lipid-linked cell surface proteins are differentially exported from the endoplasmic reticulum. Here we comment on this interdisciplinary study that highlights the role of lipid diversity and the importance of protein-lipid interactions in sorting processes at the cell membrane.
\end{abstract}

Keywords: Ceramides · GPI-anchored proteins · Protein sorting

\section{Introduction}

Eukaryotic life depends on the spatial and temporal organization of cellular membrane systems which are defined by a unique lipid composition and a specific set of proteins. The membrane signature of lipids and proteins is maintained by a complex endomembrane system that makes up the biosynthetic secretory pathway. This pathway synthesizes almost all membrane lipids and a third of the proteome in the endoplasmic reticulum to subsequently deliver them to their correct functional destinations. ${ }^{[1,2]}$ The process of directing each individual secretory protein to a specific site is known as protein sorting and is essential to preserve cellular compartmentalization and homeostasis. Indeed, a growing number of pathologies that span from genetic to neurodegenerative diseases are found to be associated with, or directly caused by sorting defects. ${ }^{[3,4]}$ Therefore, further understanding of the fundamental principles of protein sorting is a major goal for basic and biomedical research.

In the secretory pathway, proteins are transported between the different organelles in membrane-bound carriers, such as lipid vesicles. However, the sorting mechanisms by which cargo proteins are selected and captured into different transport vesicles are not completely understood. It is well established that sorting of many secretory proteins depends on specific sorting signals present on their amino acid sequence. These signals are recognized directly or indirectly by the vesicle coats, which are cytosolic protein complexes that sculpt a lipid vesicle by bending the donor membrane and, simultaneously, collect the cargo into the nascent vesicle. Nevertheless, other secretory proteins don't have known sorting signals, suggesting the existence of alternative sorting mechanisms. ${ }^{[5-7]}$

In addition to the coat-mediated sorting, the interaction of secretory proteins with the lipids of the membrane might also contribute to their sorting along the secretory pathway. Lipidomic development has shown that cell membranes contain a diverse and complex lipid composition. ${ }^{[1]}$ The precise identification of lipids has been essential to uncover the bioactive role of this special class of molecules. For instance, membrane lipids are far from just passive passengers of transport vesicles with the simple structural role of sealing the carrier container. ${ }^{[1,8]}$ They can be active players in vesicular trafficking, facilitating the recruitment of specialized cytosolic proteins of the transport machinery or increasing the membrane curvature to generate the vesicle bud. ${ }^{[9]}$ Moreover, separation of membrane lipids into different fluid phases might act as a protein sorting determinant. A lipid-based mechanism for the preferential sorting of cargo without involvement of coat proteins was initially postulated to explain the selective sorting of a specific protein from the Golgi to the apical plasma membrane in mammalian polarized epithelial cells.[10-12] This hypothetical mechanism is based on the lipid raft concept, which posits that preferential interactions by hydrogen bonding between highly saturated lipids such as sphingolipids and sterols can induce the formation of tightly packed membrane domains floating in a fluid bilayer of unsaturated glycerophospholipids. These lipid-ordered domains would recruit and laterally segregate specific proteins acting as sorting platforms for their export in selective transport carriers (Fig. 1). ${ }^{[11,13]}$

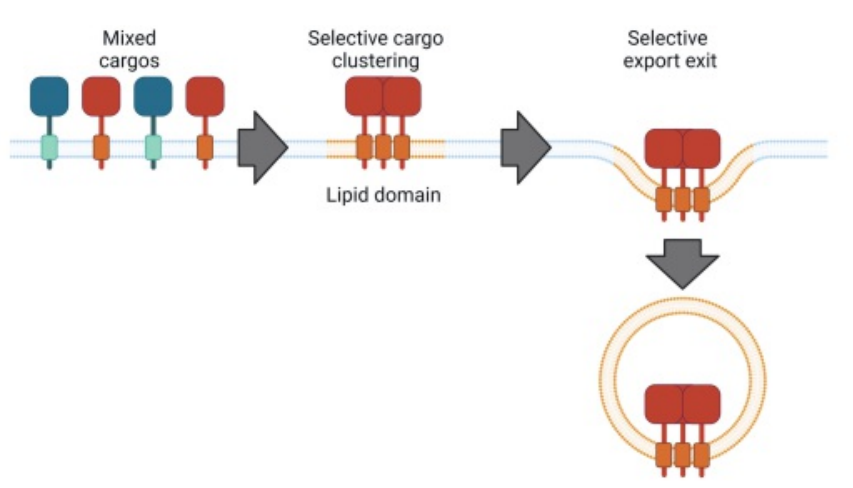

Fig 1. Lipid-based protein sorting model. Lipid-ordered domains of sphingolipids and sterols (orange) recruit and segregate specific proteins (red) for their incorporation in selective transport vesicles.

Although lipid-order phases of sphingolipids and sterols can be spontaneously generated in artificial model membranes, this mechanism is still based on indirect or inconclusive evidence in living cells. ${ }^{[14,15]}$ For that reason, lipid-based protein sorting in 
the secretory pathway is still an open question. Here, we discuss our recent work on this fundamental issue, where we specifically investigate in yeast how a diverse class of lipid-linked cell surface proteins, the glycosylphosphatidylinositol (GPI)-anchored proteins (GPI-APs), are differentially exported from the ER, the first essential step of the secretory pathway. ${ }^{[16]}$

\section{GPI-anchored Proteins as a Model to Study Lipid- dependent Protein Sorting}

GPI-APs are secretory proteins attached by a glycolipid moiety (GPI anchor) to the external leaflet of the plasma membrane, where they play a wide variety of vital physiological roles as enzymes, receptors or adhesion molecules. ${ }^{[17]}$ GPI-APs receive the GPI anchor as a posttranslational modification in the ER lumen. This modification, conserved from yeast to mammals, allows GPI-APs to travel along the secretory pathway from the ER to the plasma membrane via the Golgi compartment. The GPI anchor acts as a sorting signal that targets GPI-APs to the cell surface, conferring a unique mode of membrane association within the lumen of secretory organelles. This leads GPI-APs to be trafficked separately from transmembrane proteins along the secretory pathway. For this reason, GPI-APs constitute an exceptional model to study the relevance of protein-lipid interactions in sorting and trafficking along the secretory pathway.[18,20]

\section{The Chain Length of Ceramides Counts for Protein Sorting into Selective ER Exit Sites}

In the yeast Saccharomyces cerevisiae, GPI-APs are segregated and sorted from transmembrane cargo proteins during the ER export. ${ }^{[21,22]}$ To exit the ER, all newly synthesized secretory proteins enter into special subdomains of the ER membrane called ER exit sites (ERES), where they are packaged into the lipid vesicles that transport them to the Golgi. These vesicles are generated by polymerization of the cytosolic coat protein complex II (COPII), which locally bends the ER membrane at ERES.[23] Using an in vitro assay that reconstitutes COPII budding from the ER, we found that GPI-APs and other secretory proteins exit the ER in distinct COPII vesicles. ${ }^{[21]}$

Although the molecular bases of this sorting process were unknown, it was proposed that the underlying mechanism might require ceramide lipids. ${ }^{[19]}$ Ceramides, which consist of sphingoid long-chain base and fatty acid, serve as precursors of complex sphingolipids. ${ }^{[24]}$ In yeast, the GPI-lipid undergoes a structural remodeling at the ER after the GPI attachment to the protein. The remodeled GPI-anchor mainly incorporates a ceramide with a very long-chain saturated fatty acid of 26 carbons (C26:0). ${ }^{[17]}$ C26 ceramide, which is the major ceramide produced by yeast cells, is also synthesized in the ER and mostly exported to the Golgi by COPII vesicles. ${ }^{[25,26]}$ Furthermore, chemical and genetic experiments showed that GPI-APs had a specific ceramide requirement for their ER-to-Golgi transport in yeast. ${ }^{[27,28]}$ Biophysical studies with liposomes indicate that very long acyl chain ceramides can cluster to form ordered domains with unique physical properties. ${ }^{[29,30]}$ Based on these observations, we proposed that in the less ordered lipid environment of the yeast ER membrane formed by shorter (C16-C18) and unsaturated glycerolipids, C26 ceramides from membranes and GPI-APs take advantage of their physical properties to coalesce and cluster into ceramide-rich ordered domains. This hypothetical ceramide-dependent clustering would segregate GPI-APs from transmembrane proteins and sort them together with membrane ceramides into the same ERES and COPII vesicles. ${ }^{[19]}$

We have recently tested this hypothetical ceramide-based sorting mechanism by combining a yeast genetic system with super-resolution confocal live imaging microscopy (SCLIM), a cutting-edge microscopy technology developed by the Nakano lab (RIKEN Center for Advanced Photonics, Japan). ${ }^{[16,31]}$ This methodology allowed direct in vivo visualization of the entry of newly synthesized cargos into ERES at nanoscale dimensions. As GPI-AP we used Gas1-GFP, a yeast model GPI-AP having a C26 ceramide-based GPI-lipid moiety. ${ }^{[32]}$ We found that newly synthesized Gas1-GFP forms clusters next to specific ERES, whereas transmembrane secretory proteins distribute throughout the ER membrane. Moreover, we also observed that Gas1-GFP and transmembrane cargos selectively entered into different ERES. ${ }^{[16]} \mathrm{We}$ next tested the hypothesis that the $\mathrm{C} 26$ ceramide present in the ER membrane drives the specific clustering and sorting of GPIAPs into selective ERES. To address this goal, we took advantage of yeast genetics by working with a modified yeast strain able to produce shorter ceramides $(\mathrm{C} 18-\mathrm{C} 16)$ instead of the very long ceramides (C26) of the wild-type strain. This mutant strain named GhLag1 lacked the two endogenous yeast ceramide synthases Lag1 and Lac1 which were replaced by the ceramide synthase from the cotton GhLag1. ${ }^{[33]}$ A lipidomic approach was used to define the lipid landscape related to our biological question in the mutant strain (Fig. 2). In collaboration with the Riezman lab (University of Geneva, Switzerland) we confirmed that the membrane ceramides of the modified strain were indeed C16C18 ceramides, and we analyzed the composition of the GPI-lipid moiety of Gas1-GFP expressed in GhLag1 strain. The lipid study of the GPI-anchor involved the affinity purification of Gas1-GFP, the chemical cleavage and release of the GPI-lipid by using nitrous acid, and its analysis by electrospray ionization tandem mass spectrometry (ESI-MS/MS). ${ }^{[3]}$ For lipid species identification we used a multiple reaction monitoring (MRM) approach. By using this method, we could determine the precise GPI-lipid structure of Gas1-GFP in the GhLag1 strain. Surprisingly, we found that the Gas 1 protein expressed in the mutant strain has a C26 ceramide, the same lipid as in the wild type, ${ }^{[16,34]}$ in contrast to the ceramide composition of its cellular membranes that contains shorter ceramides (C18-C16). This fact made the GhLag1 strain an excellent candidate to study specifically the hypothetical role of the acyl chain length of membrane ceramides in ER clustering and sorting. Using SCLIM we found that when the acyl chain length of membrane ceramides decreased to C18-C16, Gas1 was unable to cluster and was rerouted to exit with other transmembrane proteins from the same ERES. Our findings showed for the first time in vivo that ceramide acyl chain length in the ER membrane is an essential player in ER clustering and sorting of GPI-APs. ${ }^{[16]}$

Coat protein complexes have been defined as one of the main drivers of protein sorting mechanisms at the early secretory pathway. ${ }^{[35]}$ Therefore, the GPI-APs clustering and sorting dependent on the membrane ceramide acyl chain could be explained because lipids are affecting the ER export protein machinery. GPI-APs use a specialized COPII system for ER export, which is actively regulated by the structural remodeling of the glycan part of the GPI anchor. ${ }^{[36,37]}$ The luminal nature of this family of proteins makes them dependent on receptors as the p24 transmembrane protein complex to ensure its recruitment in the nascent vesicle. Only when the glycan is correctly remodeled the GPI-AP is recognized by the p24 complex, which, in turn, selectively recruits Lst1, a specialized isoform of the major COPII cargo binding subunit. [38] We found that the clustering and sorting of Gas1-GFP doesn't involve the recruitment of a specialized cytosolic COPII coat. However, this process requires the presence of $\mathrm{C} 26$ ceramide in the ER membrane and the binding of the remodeled GPI-anchor by the 24 protein complex. ${ }^{[16]}$

\section{Molecular Dynamics Simulation Suggests a Mechanism for Ceramide Chain Length-based Protein Sorting}

What could be then the underlying sorting mechanism? One possibility is that p24 proteins preferentially bind through their transmembrane domains the very long chain C26 ceramide instead 


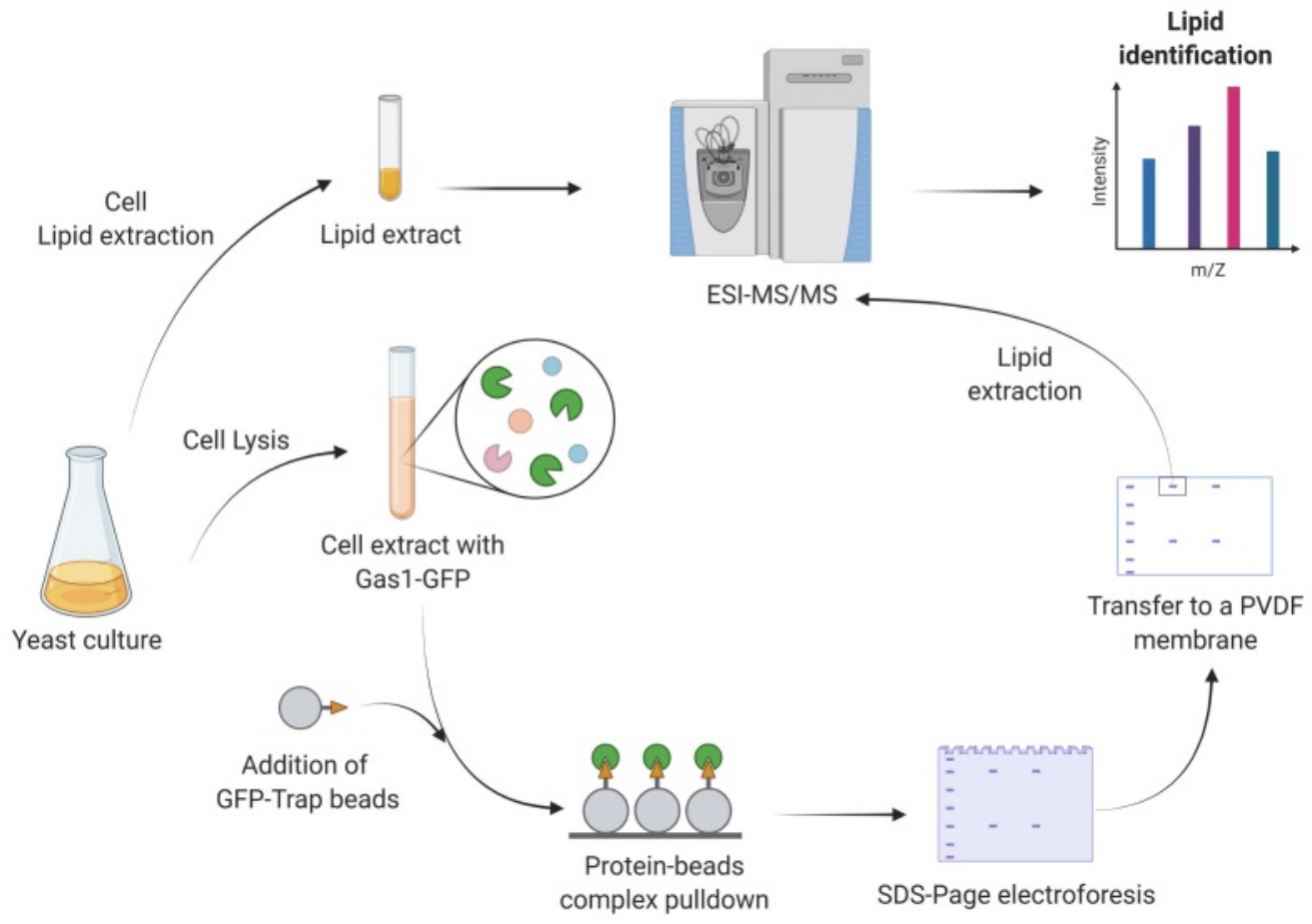

Fig 2. Overview of the lipidomic workflow used in the study by Rodriguez-Gallardo and collaborators. Yeast cells expressing Gas1-GFP were collected to obtain the cell lipid extract or to purify the GPI-AP, Gas1-GFP. Extracted lipids from the cells and the purified protein were analyzed using electrospray ionization tandem mass spectrometer.

of shorter $\mathrm{C} 18$ ceramide. To address this question, we collaborated with the Vanni lab (University of Fribourg, Switzerland). Using molecular dynamics (MD) simulations they studied the interaction between the transmembrane helix of one of the yeast p24 complex proteins, Emp24, and membrane ceramides with different acyl chain lengths. We found that both $\mathrm{C} 18$ and C26 ceramides accumulate around the cytosolic leaflet of the transmembrane helix of Emp24 causing an asymmetric distribution of ceramides in the membrane around the protein domain. Contrary to our initial hypothesis both $\mathrm{C} 26$ and $\mathrm{C} 18$ ceramides can bind Emp24 with the same preference. The underlying sorting mechanism should rely then on a distinctive biophysical property of the C26 ceramides. It has been recently shown that very-long chain C24 ceramides but not shorter $\mathrm{C} 18$ ceramides have a strong propensity to interdigitate forming interdigitated phases. ${ }^{[30]}$ In fact in liposomes this ability to interdigitate of very long chain ceramides can remodel membranes by promoting high curvature and membrane bending. ${ }^{[29]}$

Based on these findings, we propose the following mechanism to explain ceramide chain length-dependent clustering and sorting of GPI-APs into selective ERES (Fig. 3). First, the p24 complex binds multivalently C26 ceramide-based GPI-APs in the luminal leaflet and, concomitantly, accumulates C26 membrane ceramides in the cytosolic leaflet. The local concentration of C26 ceramides in both leaflets of the ER membrane around the p24 proteins would lead them to interdigitate forming an interdigitated phase, which in turn, would promote protein clustering and membrane curvature, leading to GPI-AP segregation into selective ERES, which also prefers highly curved zones of the ER membrane. ${ }^{[39]}$ A partial interdigitation between C26 ceramides around the p24 proteins is predicted to locally increase the thickness of the ER membrane. Indeed, a recent study by the Barral lab (ETH Zurich) has described genetically encoded fluorescent reporters visualizing local variations in bilayer thickness. ${ }^{[40]}$ These reporters or biosensors were used to show that $\mathrm{C} 26$ membrane ceramides promote the formation of discrete domains of increased bilayer thickness in the yeast ER. Therefore, these biosensors could be used to test whether the discrete domains formed by clustered
GPI-APs adjacent to specific ERES are thicker than the bulk of the ER membrane. In this case, thickness could act also as sorting determinant by generating hydrophobic mismatches.

Contrary to yeast, in mammalian cells, most of GPI-APs have an ether lipid-based GPI anchor. Both lipids are structurally different, which raises the question of whether the mechanism proposed in yeast might be also conserved in mammals. Interestingly, despite the differences in the chemical structure of the ether lipids and very long acyl chain ceramides, a recent study found that both lipids evolutionarily share some similar physicochemical properties and functions. ${ }^{[41]}$ Thus, we could postulate that the ether lipid of GPI anchors in mammalian cells might function similarly to the very long acyl chain ceramide of GPI anchors in yeast. The ether lipid moiety could associate with very long-chain ceramides present in the ER membrane promoting clustering and sorting of GPI-APs during ER export. This model that implies a tight correlation between GPI-APs and long-chain membrane ceramides is supported by previous observations in mammalian cells and yeast, where GPI anchor synthesis is required for the biosynthetic transport to the Golgi of very long acyl chain ceramides.[42,43] Therefore, an evolutionarily conserved mechanism appears to operate for selective co-transport of GPI-APs and very long acyl chain ceramides in the same transport vesicles.

\section{Conclusions}

Sorting mechanisms are of vital importance to keep cell compartmentalization and functions. In this review we summarize how interdisciplinary research that combines genetic engineering, chemistry, lipidomics, super-resolution confocal live imaging microscopy and molecular dynamic simulations has been highly useful to decipher the role of very long chain ceramides in the sorting mechanism of essential secretory proteins as the GPI-APs. Contrary to the classical lipid-based protein sorting model, our results suggest that it is not the lipid alone but transmembrane proteins who initiate sorting by locally reorganizing and concentrating specific lipids. We propose that the biophysical properties associated with the concentrated lipids around the transmembrane 
Fig 3. Model proposed for ceramide chain length-based GPI-AP sorting at the ER (A) Left, fluorescent micrograph of a yeast cell with normal C26 membrane ceramides expressing the GPI-AP Gas1-GFP. Gas1-GFP is concentrated into clusters at the ER membrane (white filled arrowhead). Scale bar, $5 \mu \mathrm{m}$. Right, schematic representation showing how the p24 complex promotes local phase separation and acyl chain interdigitation by concentrating C26 membrane ceramides in the cytosolic leaflet and C26 ceramide-based GPIAPs in the luminal leaflet of the ER membrane. This leads to protein clustering and membrane curvature which segregates GPI-APs into discrete zones next to selective ERES. (B) Left, fluorescent micrograph of a mutant yeast cell with shorter $\mathrm{C} 18-\mathrm{C} 16$ membrane ceramides expressing Gas1-GFP. Unclustered Gas1GFP is distributed throughout the ER membrane displaying the ERcharacteristic nuclear ring staining (open arrowhead). Scale bar, $5 \mu \mathrm{m}$. Right, schematic representation showing how decreasing the acyl chain length of cellular ceramide in the membrane from C26 to C18-C16 prevents acyl chain interdigitation, which disrupts clustering of GPI-APs and reroutes them to exit the ER with transmembrane proteins via the same ERES.
A
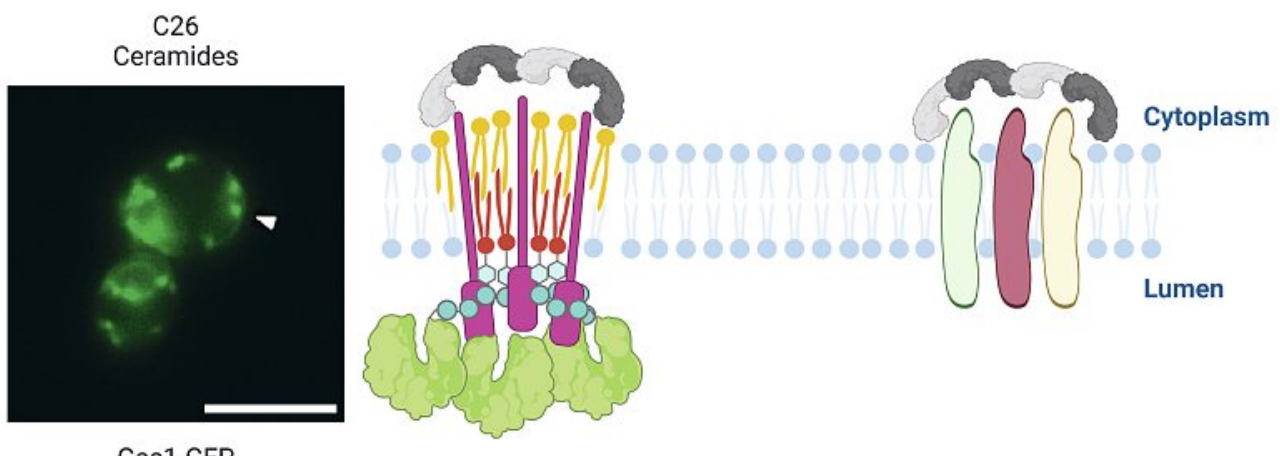

Gas1-GFP

B

C16-18

Ceramides

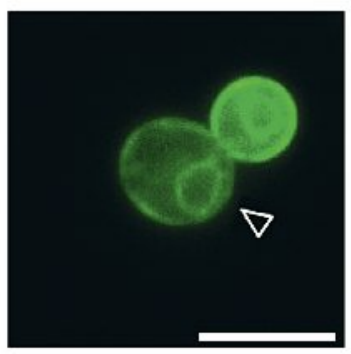

Gas1-GFP

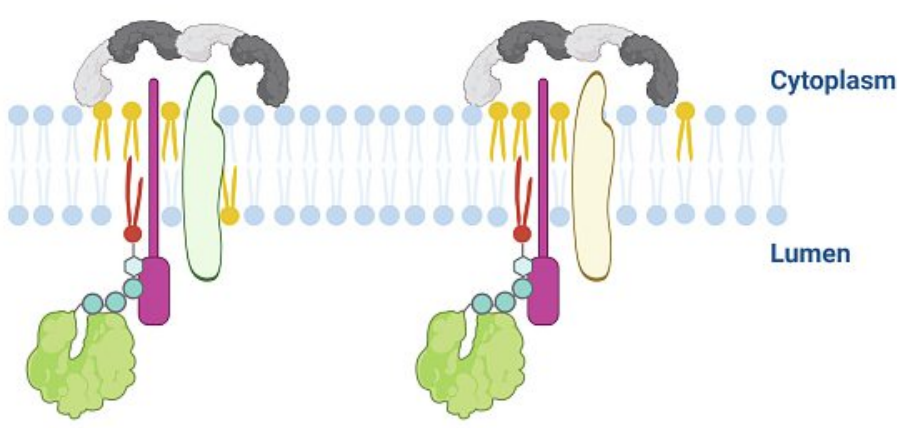

Glycerophospholipid C18

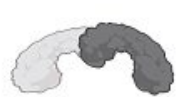

COPII coat

Ceramide C26

GPI-APS
1

Ceramide $\mathrm{C} 18-\mathrm{C} 16$ domains eventually drive lateral segregation and sorting of proteins. According to this model, proteins might recruit the appropriate lipids exploiting their specific emergent biophysical properties to perform different functions within the cell. This would explain how diverse lipid composition of the membrane makes sense in cell physiology. Therefore, understanding the local mutual influence of lipids and proteins will be key to comprehend eukaryotic life.

\section{Acknowledgements}

The authors are grateful for funding from the FEDER/Ministerio de Ciencia, Innovación y Universidades - Agencia Estatal de Investigación/ PID2020-119505GB-I00 and BFU2017-89700-P to Manuel Muñiz. The figures were created with BioRender.com

Received: September 30, 2021

[1] T. Harayama, H. Riezman, Nat. Rev. Mol. Cell Biol. 2018, 19, 281, https://doi.org/10.1038/nrm.2017.138.

[2] J. S. Bonifacino, B. S. Glick, Cell 2004, 116, 153, https://doi.org/10.1016/S0092-8674(03)01079-1.

[3] R. Di Martino, L. Sticco, A. Luini, FEBS Lett. 2019, 593, 2306, https://doi.org/10.1002/1873-3468.13572

[4] X. Wang, T. Huang, G. Bu, H. Xu, Mol. Neurodegener. 2014, 9, 31, https://doi.org/10.1186/1750-1326-9-31.
[5] A. D. Gillon, C. F. Latham, E. A. Miller, Biochim. Biophys. Acta 2012, 1821, 1040, https://doi.org/10.1016/j.bbalip.2012.01.005

[6] S. Springer, P. Malkus, B. Borchert, U. Wellbrock, R. Duden, R. Schekman, Traffic 2014, 15, 531, https://doi.org/10.1111/tra.12157

[7] C. Barlowe, Trends Cell Biol. 2003, 13, 295, https://doi.org/10.1016/s0962-8924(03)00082-5.

[8] K. Funato, H. Riezman, M. Muñiz, Biochim. Biophys. Acta Mol. Cell Biol. Lipids 2020, 1865, 158453, https://doi.org/10.1016/j.bbalip.2019.04.013.

[9] A. Melero, N. Chiaruttini, T. Karashima, I. Riezman, K. Funato, C. Barlowe, H. Riezman, A. Roux, Curr. Biol. 2018, 28, 1950, https://doi.org/10.1016/j.cub.2018.04.076.

[10] K. Simons, G. van Meer, Biochemistry 1988, 27, 6197, https://doi.org/10.1021/bi00417a001

[11] M. A. Surma, C. Klose, K. Simons, Biochim. Biophys. Acta 2012, 1821, 1059, https://doi.org/10.1016/j.bbalip.2011.12.008.

[12] K. Simons, E. Ikonen, Nature 1997, 387, 569, https://doi.org/10.1038/42408,

[13] K. Simons, J. L. Sampaio, Cold Spring Harb. Perspect. Biol. 2011, 3, a004697, https://doi.org/10.1101/cshperspect.a004697.

[14] E. Sezgin, I. Levental, S. Mayor, C. Eggeling, Nat. Rev. Mol. Cell Biol. 2017, 18, 361, https://doi.org/10.1038/nrm.2017.16

[15] I. Levental, K. R. Levental, F. A. Heberle, Trends Cell Biol. 2020, 30, 341, https://doi.org/10.1016/j.tcb.2020.01.009.

[16] S. Rodriguez-Gallardo, K. Kurokawa, S. Sabido-Bozo, A. Cortes-Gomez, A. Ikeda, V. Zoni, A. Aguilera-Romero, A. M. Perez-Linero, S. Lopez, M. Waga, M. Araki, M. Nakano, H. Riezman, K. Funato, S. Vanni, A. Nakano, M. Muñiz, Sci. Adv. 2020, 6, https://doi.org/10.1126/sciadv.abc6250.

[17] T. Kinoshita, M. Fujita, J. Lipid Res. 2016, 57, 6 , https://doi.org/10.1194/jlr.R063313.

[18] S. Mayor, H. Riezman, Nat. Rev. Mol. Cell Biol. 2004, 5, 110 https://doi.org/10.1038/nrm1309 
[19] M. Muñiz, C. Zurzolo, J. Cell Sci. 2014, 127, 2793, https://doi.org/10.1242/jcs.148056.

[20] M. Muñiz, H. Riezman, J. Lipid Res. 2016, 57, 352, https://doi.org/10.1194/jlr.R062760.

[21] M. Muñiz, P. Morsomme, H. Riezman, Cell 2001, 104, 313, https://doi.org/10.1016/S0092-8674(01)00215-X.

[22] G. A. Castillon, R. Watanabe, M. Taylor, T. M. Schwabe, H. Riezman, Traffic 2009, 10, 186, https://doi.org/10.1111/j.1600-0854.2008.00857.x.

[23] K. Kurokawa, A. Nakano, J. Biochem. 2019, 165, 109, https://doi.org/10.1093/jb/mvy080.

[24] A. H. Futerman, H. Riezman, Trends Cell Biol. 2005, 15, 312, https://doi.org/10.1016/j.tcb.2005.04.006.

[25] C. S. Ejsing, J. L. Sampaio, V. Surendranath, E. Duchoslav, K. Ekroos, R. W. Klemm, K. Simons, A. Shevchenko, Proc. Natl Acad. Sci. USA 2009, 106, 2136, https://doi.org/10.1073/pnas.0811700106

[26] K. Funato, H. Riezman, J. Cell Biol. 2001, 155, 949, https://doi.org/10.1083/jcb.200105033.

[27] A. Horvath, C. Sütterlin, U. Manning-Krieg, N. R. Movva, H. Riezman, EMBO J. 1994, 13, 3687

[28] C. Sütterlin, T. L. Doering, F. Schimmöller, S. Schröder, H. Riezman, J. Cell Sci. 1997, 110 ( Pt 21), 2703.

[29] S. N. Pinto, L. C. Silva, A. H. Futerman, M. Prieto, Biochim. Biophys. Acta 2011, 1808, 2753, https://doi.org/10.1016/j.bbamem.2011.07.023.

[30] A. E. Ventura, A. R. P. Varela, T. Dingjan, T. C. B. Santos, A. Fedorov, A. H. Futerman, M. Prieto, L. C. Silva, Biochim. Biophys. Acta Biomembr. 2020, 1862, 183400, https://doi.org/10.1016/j.bbamem.2020.183400.

[31] K. Kurokawa, M. Ishii, Y. Suda, A. Ichihara, A. Nakano, Methods Cell Biol. 2013, 118, 235, https://doi.org/10.1016/B978-0-12-417164-0.00014-8.

[32] T. Yoko-O, D. Ichikawa, Y. Miyagishi, A. Kato, M. Umemura, K. Takase, M. Ra, K. Ikeda, R. Taguchi, Y. Jigami, Mol. Microbiol. 2013, 88, 140, https://doi.org/10.1111/mmi.12175.

[33] S. Epstein, G. A. Castillon, Y. Qin, H. Riezman, Mol. Microbiol. 2012, 84, 1018, https://doi.org/10.1111/j.1365-2958.2012.08087.x.

[34] A. Aguilera-Romero, S. Sabido-Bozo, S. Lopez, A. CortesGomez, S. Rodriguez-Gallardo, A. M. Perez-Linero, I. Riezman, H. Riezman, M. Muñiz, PLoS One 2021, 16, e0256184, https://doi.org/10.1371/journal.pone.0256184.
[35] J. Dancourt, C. Barlowe, Апnи. Rev. Biochem. 2010, 79, 777, https://doi.org/10.1146/annurev-biochem-061608-091319.

[36] J. Manzano-Lopez, A. M. Perez-Linero, A. Aguilera-Romero, M E. Martin, T. Okano, D. V. Silva, P. H. Seeberger, H. Riezman, K. Funato, V. Goder, R. E. Wellinger, M. Muñiz, Curr. Biol. 2015, 25, 152 , https://doi.org/10.1016/j.cub.2014.11.039

[37] M. Nakano, S. Sabido-Bozo, K. Okazaki, A. Aguilera-Romero, S. RodriguezGallardo, A. Cortes-Gomez, S. Lopez, A. Ikeda, K. Funato, M. Muñiz, PLoS One 2021, 16, e0257435, https://doi.org/10.1371/journal.pone.0257435.

[38] E. A. Miller, T. H. Beilharz, P. N. Malkus, M. C. Lee, S. Hamamoto, L. Orci, R. Schekman, Cell 2003, 114, 497, https://doi.org/10.1016/S0092-8674(03)00609-3.

[39] M. Okamoto, K. Kurokawa, K. Matsuura-Tokita, C. Saito, R. Hirata, A. Nakano, J. Cell Sci. 2012, 125, 3412, https://doi.org/10.1242/jcs.100065.

[40] R. Prasad, A. Sliwa-Gonzalez, Y. Barral, Sci. Adv. 2020, 6, https://doi.org/10.1126/sciadv.aba5130.

[41] N. Jiménez-Rojo, M. D. Leonetti, V. Zoni, A. Colom, S. Feng, N. R. Iyengar, S. Matile, A. Roux, S. Vanni, J. S. Weissman, H. Riezman, Curr. Biol. 2020, 30, P3775, https://doi.org/10.1016/j.cub.2020.07.059.

[42] K. Kajiwara, R. Watanabe, H. Pichler, K. Ihara, S. Murakami, H. Riezman, K. Funato, Mol. Biol. Cell 2008, 19, 2069, https://doi.org/10.1091/mbc.e07-08-0740.

[43] U. Loizides-Mangold, F. P. David, V. J. Nesatyy, T. Kinoshita, H. Riezman, J. Lipid Res. 2012, 53, 1522, https://doi.org/10.1194/jlr.M025692.

\section{License and Terms}

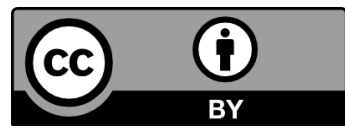

This is an Open Access article under the terms of the Creative Commons Attribution License CC BY 4.0. The material may not be used for commercial purposes.

The license is subject to the CHIMIA terms and conditions: (http:// chimia.ch/component/sppagebuilder/?view=page\&id=12).

The definitive version of this article is the electronic one that can be found at https://doi.org/10.2533/chimia.2021.1026 